\title{
ENHANCED ELECTROCHEMICAL ENERGY STORAGE BY NANOSCOPIC DECORATION OF ENDOHEDRAL AND EXOHEDRAL CARBON WITH VANADIUM OXIDE VIA ATOMIC LAYER DEPOSITION
}

\author{
Simon Fleischmann, ${ }^{1,2}$ Nicolas Jäckel, ${ }^{1,2}$ Marco Zeiger, ${ }^{1,2}$ \\ Benjamin Krüner, ${ }^{1,2}$ Ingrid Grobelsek, ${ }^{1}$ Petr Formanek, ${ }^{3}$ \\ Soumyadip Choudhury, ${ }^{1,3}$ Daniel Weingarth, ${ }^{1}$ Volker Presser ${ }^{1,2,}{ }^{*}$ \\ 2 Department of Materials Science and Engineering, Saarland University, 66123 Saarbrücken, Germany \\ 3 Leibniz-Institut für Polymerforschung Dresden e. V., 01069 Dresden, Germany
}

1 INM - Leibniz Institute for New Materials, 66123 Saarbrücken, Germany

* Corresponding author's eMail: volker.presser@leibniz-inm.de 


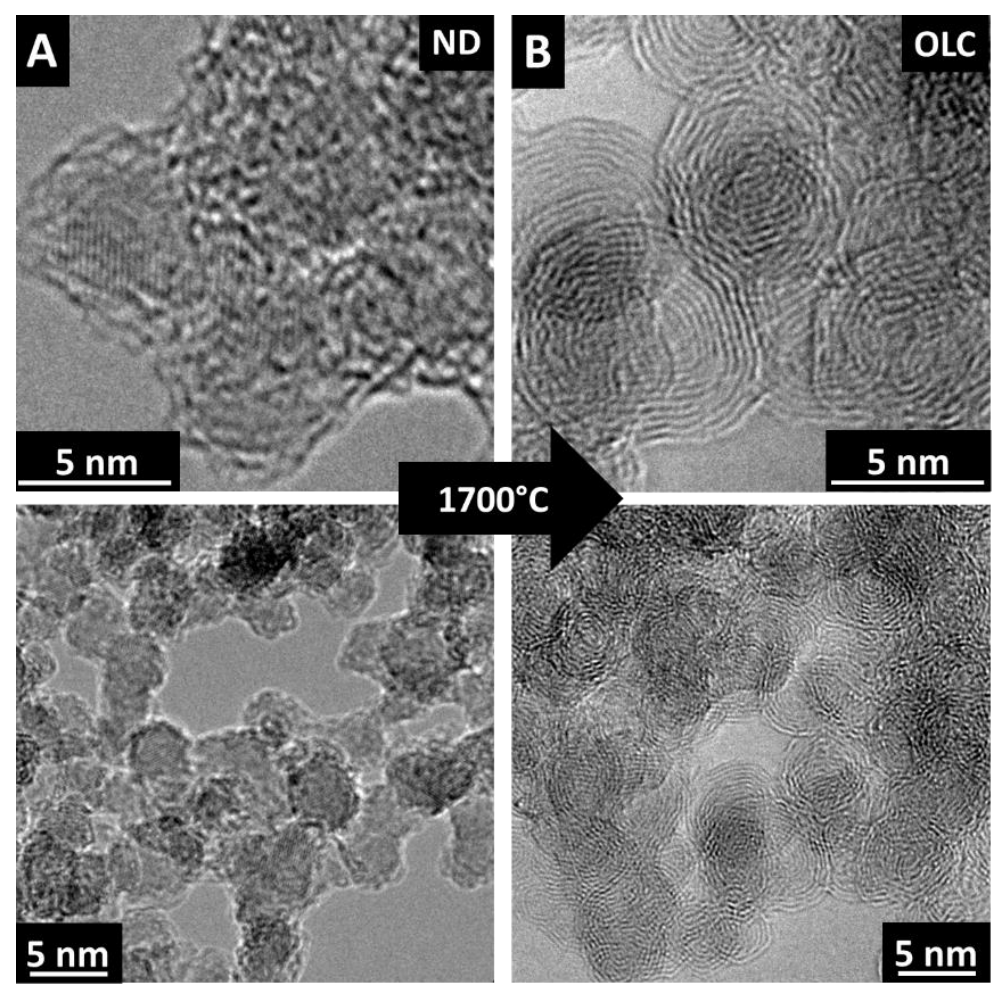

Fig. S1: Transmission electron micrographs of nanodiamond precursor (ND) (A) and the resulting carbon onions (B) after thermal annealing in argon atmosphere at $1700^{\circ} \mathrm{C}$ for $1 \mathrm{~h}$. The top and bottom rows show different magnifications. 


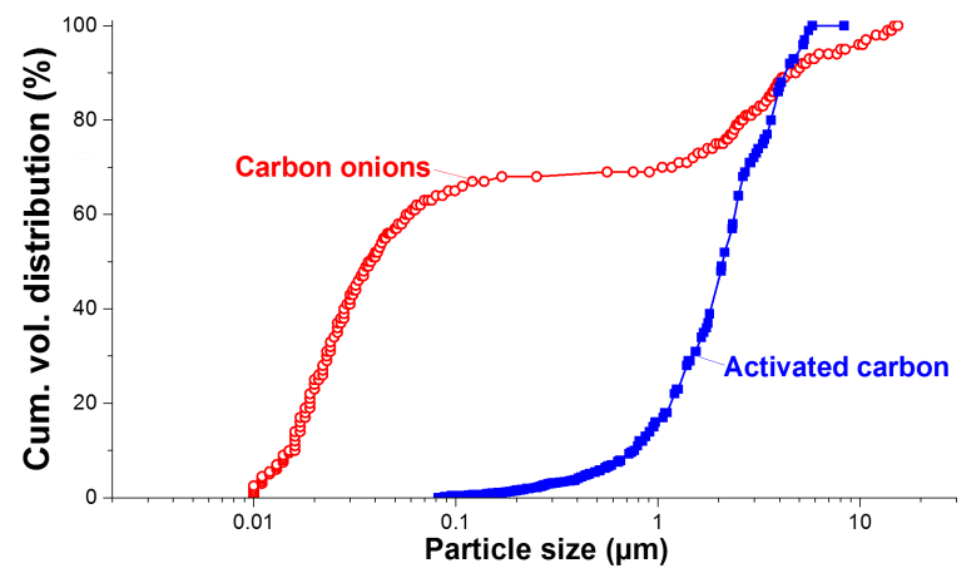

Fig. S2: Cumulative volume distribution of carbon onions and activated carbon particles plotted against the particle size. Measured by centrifugal sedimentation analysis in ethanol, adapted from Ref. ${ }^{23}$. 

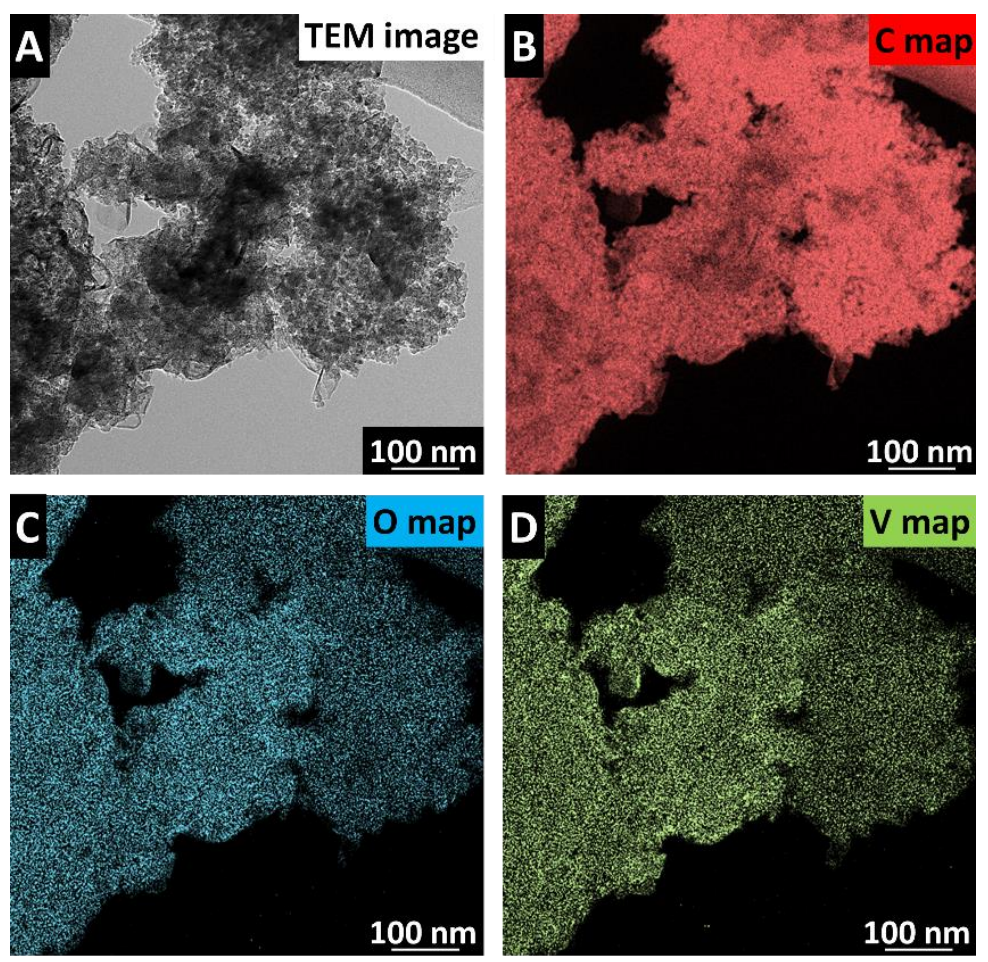

Fig. S3: Transmission electron micrograph of carbon onion particles coated with 100 ALD cycles (A), and the corresponding EFTEM maps of carbon (B), oxygen (C), and vanadium (D). 

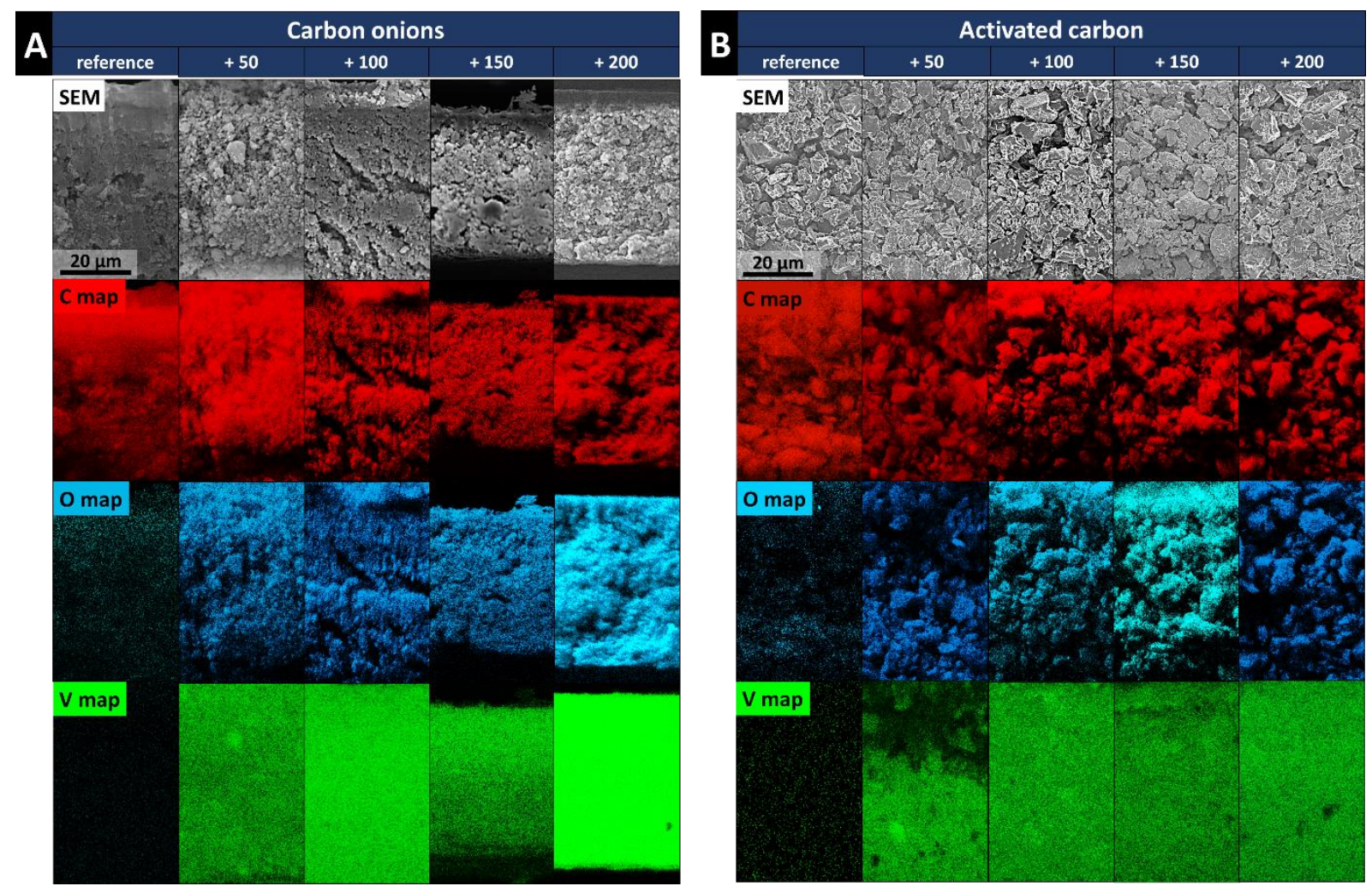

Fig. S4: Cross sectional EDX mappings of carbon onion (A) and activated carbon (B) hybrid electrodes. The element distribution of carbon ( $C$ map), oxygen ( $O$ map), and vanadium ( $V$ map) is illustrated across the whole electrode cross section, including the original SEM image in the top row. For the analysis, the $\mathrm{C}_{\mathrm{K}^{-}}, \mathrm{O}_{\mathrm{K} \alpha^{-}}$, and $\mathrm{V}_{\mathrm{L} \alpha^{-}}$-lines were employed. 

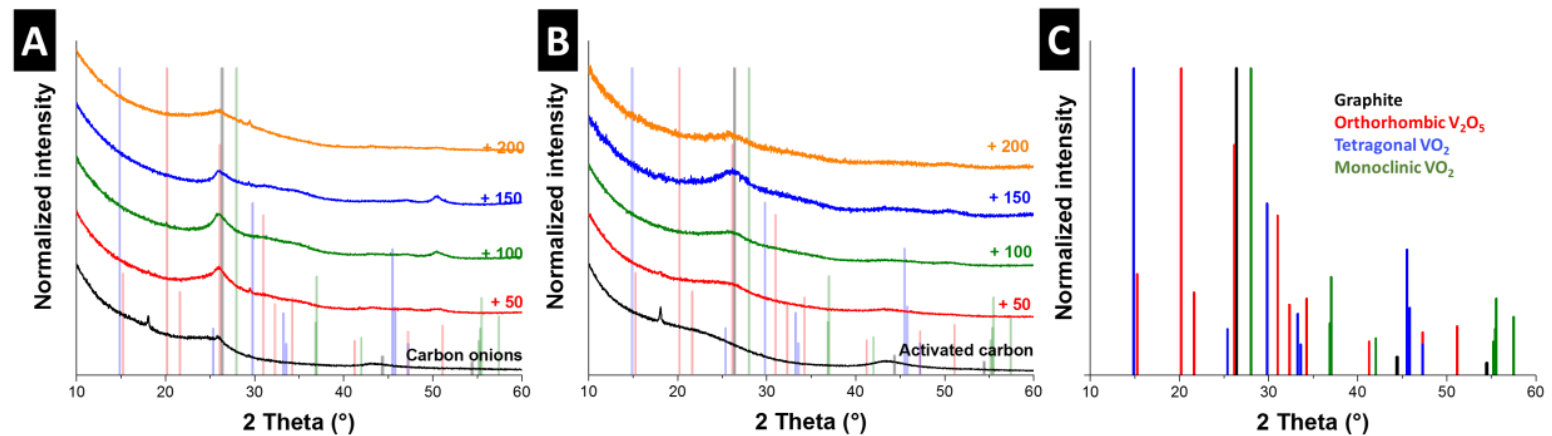

Fig. S5: X-ray diffractograms of carbon onion (A) and activated carbon (B) hybrid electrodes. The ideal peak positions from the powder diffraction files for orthorhombic $\mathrm{V}_{2} \mathrm{O}_{5}$ (PDF 41-1426), tetragonal $\mathrm{VO}_{2}$ (PDF 42-0876), monoclinic $\mathrm{VO}_{2}$ (PDF 44-0353), and graphite (PDF 41-1487) are given in (C) and, with transparency, visible in the background of (A) and (B). The small sharp peaks for carbon onions and activated carbons at $18^{\circ} 2 \theta$ is associated with PTFE (which is the polymer binder used for electrode manufacturing). 


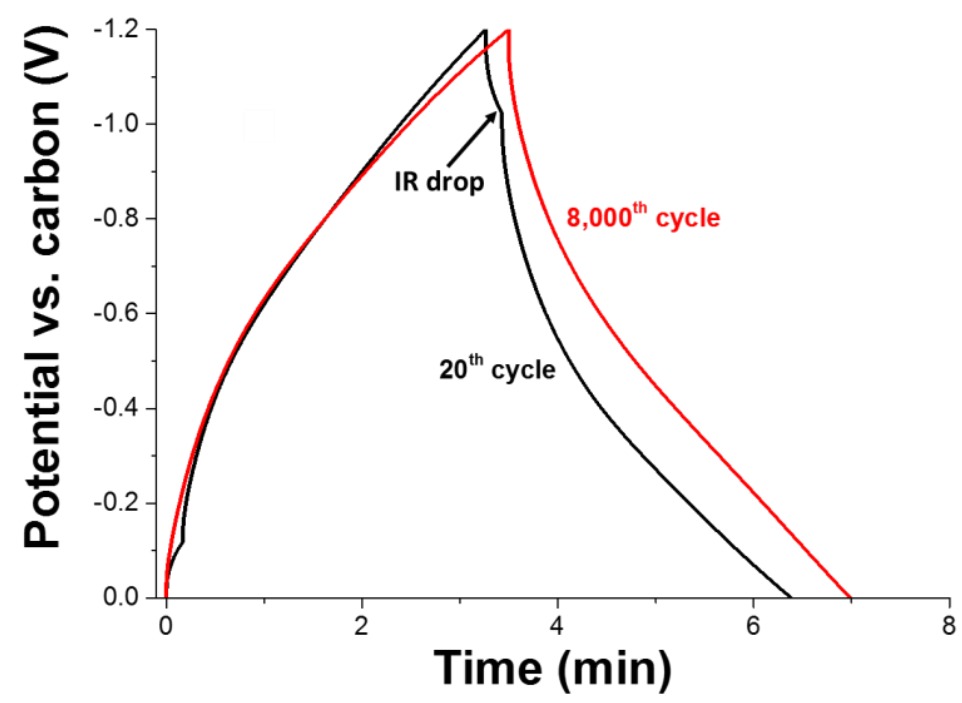

Fig. S6: Galvanostatic charge-discharge profiles of $A C+100$ during stability testing. The black line shows the presence of an IR drop in the beginning of the stability test $\left(20^{\text {th }}\right.$ cycle), while the red line $\left(8,000^{\text {th }}\right.$ cycle) shows no IR drop. 